\title{
Beitrag zur Kenntnis der Feldspäte der Tessiner Pegmatite
}

\section{Doctoral Thesis}

Author(s):

Paraskevopoulos, Georg Michel

Publication date:

1953

Permanent link:

https://doi.org/10.3929/ethz-a-000088936

Rights / license:

In Copyright - Non-Commercial Use Permitted 


\title{
Beitrag zur Kenntnis der Feldspäte der Tessiner Pegmatite
}

\author{
VON DER \\ EIDGENÖSSISCHEN TECHNISCHEN \\ HOCHSCHULE IN ZU்RICH \\ ZUR ERLANGUNG \\ DER WÜRDE EINES DOKTORS DER \\ NATURWISSENSCHAFTEN \\ GENEHMIGTE \\ PROMOTIONSARBEIT \\ VORGELEGT VON \\ GEORG M. PARASKEVOPOULOS \\ aus Athen (Griechenland)
}

Referent: Herr Prof. Dr. P. Niggli $\uparrow$

Korreferent: Herr Prof. Dr. C. Burri

Springer-Verlag Wien/1953 
Das Auftreten von Dumortierit in schweizerischen Pegmatiten ist auch von früheren Forschungen her bekannt. So z. B. haben E. Hugi und H. Hirschi (37) Dumortierit mit Turmalin und Granat in den Pegmatiten des Bergells in den südlichen Schweizeralpen gefunden mit $2 \mathrm{~V}_{\alpha}=30^{\circ}$. Mittelholzer (54) erwähnt ein Vorkommen von Dumortierit zusammen mit Disthen in den Pegmatiten von Castione, wobei Dumortierit $2 \mathrm{~V}_{\alpha}=38^{\circ}$ besitzt. Der Pleochroismus war in diesem Fall

$$
\begin{aligned}
& n_{\alpha} \text { tief rotviolett } \\
& n_{\beta} \text { blaurosa } \\
& n_{\gamma} \text { farblos. }
\end{aligned}
$$

Beryll. Dieses Mineral wurde in den Pegmatiten von Ponte Brolla und Maggiatal gefunden. Es ist aber auch aus anderen Pegmatitvorkommen bekannt. Unser Beryll hat eine grüne bis grünblaue Farbe, zeigt aber auch seltener hellere Farbe. Gut ausgebildete Kristalle sind spärlich.

Anhangsweise seien auch die von $F$. de Quervain in den Pegmatiten von Brissago gefundene Uranpechblende und die Fe-Mn-Phosphate erwähnt, über die man Näheres in der Originalarbeit finden kann.

\section{Zusammenfassung, Einteilung der Pegmatite und Diskussion über ihre Entstehung.}

Im folgenden sei kurz erwähnt, wie sich die Autoren, die sich mit den Tessiner Pegmatiten beschäftigten, über die Gliederung bzw. die Entstehung dieser Gesteine äußerten.

De Quervain (63) beschreibt von Brissago nur Albitpegmatite, in denen selten Mikroklin vorkommt und dies in sehr kleinen Mengen (Verdrängung durch Albit).

Urban (84) unterschied - wie früher erwähnt - in der Umgebung von Bellinzona eine granit-aplitische und eine quarzdioritische Injektion. Dazu bemerkt Mittelholzer (54), daB die als quarz• dioritisch bezeichneten Injektionen ursprünglich auch granitpegmatitisch gewesen sein können, sofern eine mehr oder weniger starke Stoffaufnahme stattgefunden habe.

Mittelholzer (54, S. 92) unterscheidet zwei extreme Typen, 1. Mikroklinpegmatite, wobei außer dem Mikroklin + saurer Plagioklas vorhanden ist, und 2. Albitpegmatite, bei denen neben dem Albit $\perp$ Mikroklin anzutreffen ist. In beiden Fällen findet man neben den Feldspäten und Quarz auch Granat, Turmalin, Muskovit und Biotit (von seltenen Mineralien abgesehen). Dem ersten Typus gehören die Pegmatite der Zone von Bellinzona und dem zweiten der große Pegmatit von Gordola-Ponte della Torretta an. Mittelholzer schreibt 
aber weiter: „Beide Gruppen (Albit- und Mikroklin-Pegmatite) sind durch zahlreiche Übergänge, oft sogar in einem Gang, miteinander verbunden" $(54$, S. 93).

Kern (43) konnte im Centovalli und Pedemonte Kaliumfeldspatpegmatite mit untergeordneten Mengen von saurem Plagioklas $(0$ bis 15\% An) und Plagioklaspegmatite ohne Kaliumfeldspat und mit Plagioklas zwischen 15 und $45 \%$ An (vorwiegend aber mit Oligoklas) unterscheiden.

Walter (86) hat beiläufig die Pegmatite von Verbano-Ivrea untersucht und meint, daß die grobkörnigen Pegmatite der Kinzigitzone mit den Albitpegmatiten von Mittelholzer aus der Zone von Bellinzona zusammengehören. Bei den feinkörnigen der gleichen Zone kann er nicht entscheiden, ob sie den Mikroklinpegmatiten der Zone von Bellinzona oder den alten, präalpinen Pegmatiten zugezählt werden müssen. In der Ivrea-Zone findet er in den basischen Augit- und Hornblendegesteinen Plagioklaspegmatite, deren Plagioklas ähnlichen An-Gehalt aufweist wie der An-Gehalt der Plagioklase der Nebengesteine. Walter betrachtet diese Pegmatite - mindestens zum Teil - eher als Restlösungen der basischen Magmen, er vermutet aber, da $B$ auch andere Pegmatite auftreten, die mit denen der Kinzigitzone verwandt sind. In der Zone von Arcegno erwähnt der gleiche Autor Pegmatite mit Mikroklin und saurem Plagioklas, Muskovit, Granat, spärlichem Biotit oder Chlorit und selten Turmalin.

Kündig (46) hat neben normalen Pegmatiten mit Beryll und Turmalin auch Plagioklas-Turmalinpegmatite beschrieben, die zur Eklogit-Amphibolitfacies der Ophiolithe des Passo di Sanano gehören.

Nach unseren Untersuchungen läßt die Zusammensetzung der Feldspäte die Pegmatile des speziellen Untersuchungsgebietes in drei Gruppen einteilen.

1. Pegmatite mit Kaliumfeldspat und Albit bis Oligoklas $(0$ bis $15 \%$ An und seltener bis $20 \%$ An).

2. Pegmatite mit Kaliumfeldspat und Oligoklas bis Andesin (20 bis $34 \%$ An).

3. Plagioklaspegmatite mit basischem Oligoklas bis Andesin (22 bis $35 \%$ An) und sehr spärlichem oder fehlendem Kaliumfeldspatgehalt.

Am häufigsten trafen wir die erste Gruppe an, dann der Reihe nach die zweite und dritte. Die letzte Kategorie ist relativ selten. Es ist aber zu bemerken, daB die ersten zwei Gruppen nicht streng voneinander getrennt werden können, weil dazwischen Übergänge existieren. Im weiteren sind Fälle beobachtet worden, bei denen der 
Kaliumfeldspat gegenüber dem Plagioklas sehr stark überwiegt neben anderen mit umgekehrten Mengenverhältnissen. Beide Varianten können sogar im gleichen Gang vorkommen. Dies beruht wohl auf der Tatsache, daß die Pegmatitlösungen sehr kalireich waren, so daß mit beginnender Kristallisation zuerst Kaliumfeldspat ausgeschieden und manchmal in beträchtlichen Mengen angehäuft wurde. Die Restlösungen wurden dadurch an $\mathrm{Na}_{2} \mathrm{O}$ angereichert und die einsetzende Kristallisation von Plagioklas hatte zur Folge, daß der letztere den Kaliumfeldspat verdrängte oder ihn quantitativ zu überwiegen vermochte. Die Verdrängung des Kaliumfeldspates ist ein sehr verbreitetes Phänomen. Fast alle Pegmatite lassen dieses Phänomen erkennen und Fälle einer sehr starken Verdrängung sind nicht selten. Die Plagioklaspegmatite selbst bewahren oft noch sehr spärliche Reste von Kaliumfeldspat, die dann als Zeugen einer allzu starken, auch hier vorhanden gewesenen Verdrängung dieses Minerals durch Plagioklas anzusehen sind.

So gehören die Albitpegmatite de Quervains, die Mikroklinpegmatite und Albitpegmatite Mittelholzers und die Kaliumfeldspatpegmatite sowie die sauren Glieder der Plagioklaspegmatite Kerns meiner ersten oder zweiten Gruppe, vielleicht ein und derselben Differentiationsfolge an, mit zunehmendem Na-Gehalt in den Spätphasen. Nebenher sei gesagt, daß bei Brissago auch Pegmatite gefunden wurden, die neben Albitoligoklas Kaliumfeldspat führen.

Mittelholzers Mikroklinpegmatite und Kerns Kaliumfeldspatpegmatite lassen sich meiner ersten Gruppe einordnen. Die Plagioklaspegmatite von Kern müssen schließlich in unsere dritte Gruppe fallen. Die sauren Glieder der Plagioklaspegmatite gehören den extremen Typen meiner zweiten Gruppe an und bilden den Übergang zur dritten.

Der Grund, daß ich diese Klassifikation vorziehe, liegt in einer engen Beziehung der Entstehungsart der ersten zwei Gruppen, die meiner Meinung nach durch eine allmähliche Differentiation der pegmatitischen Restlösungen auseinander entstanden sind. Aus den oft großen Mengen des Kaliumfeldspates, aus der überall verbreiteten Verdrängung von seiten des Plagioklases her, muß man an einen ursprünglich erheblichen Gehalt von $\mathrm{K}_{2} \mathrm{O}$ in den ältesten Restlösungen denken. Anderseits wurde schon erwähnt, daß für die ersten zwei Gruppen Übergänge von der einen in die andere Gruppe beobachtet wurden und daß auch das Verhältnis Kaliumfeldspat: : Plagioklas sogar im gleichen Gang sehr stark variieren kann. Ana$\log$ hat auch Mittelholzer aus Bellinzona berichtet, daß zwischen seinen Mikroklinpegmatiten einerseits und Albitpegmatiten anderseits zahlreiche UUbergänge, sogar in einem Gang, existieren können. 
Von diesen Betrachtungen ausgehend, stelle ich mir die Entstehung der Pegmatite der ersten und der zweiten Gruppe folgendermaßen vor. Zu Beginn der Injektionen waren die Lösungen sehr kalireich. Es wurde zuerst Kaliumfeldspat ausgeschieden und nach bestimmter Zeit setzte auch die Kristallisation von Plagioklas ein. nachdem die Restlösungen an $\mathrm{Na}_{2} \mathrm{O}$ ausgereichert worden waren.

Die zuerst kristallisierten

Plagioklase waren Oligoklase bis saure Andesine, und mit ihrer Ausscheidung begann auch die Verdrängung des Kaliumfeldspates. Nach der Kristallisation dieser Pegmalite, die bereits zur zweiten Gruppe gehören, ging in der Tiefe die Differentiation der Restlösungen weiter, so daß die neu ausgeschiedenen Plagioklase immer saurer wurden. Dann folgten Pegmatite mit saurem Oligoklas und die Gesteine gingen sukzessiv von der zweiten Gruppe in die erste Gruppe über. Der Proze 6 ging nun in dieser Richtung weiter, bis auch die sauerslen Glieder der ersten Gruppe entstanden waren, wobei als Plagioklas nur sau-

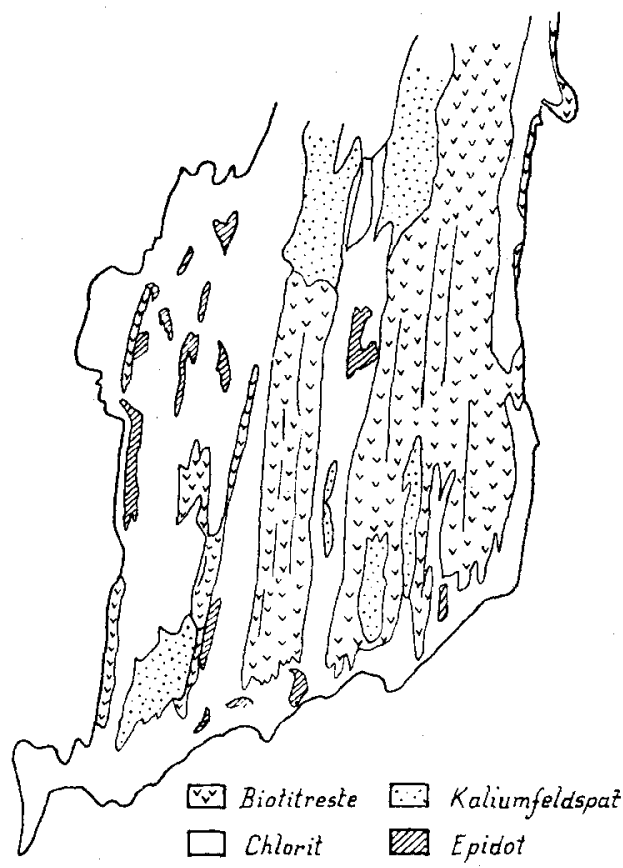

Abb. 25. Bildung von Kaliumfeldspat durch Umwandlung des Biotites. Schliff $\triangle_{2}$. rer Oligoklas und Albit ausgeschieden wurden. In den letzten Kristallisationsprodukten sind Albitpegmatite gebildet worden, bei denen der Kaliumfeldspat manchmal slark zurücktreten kann.

Mit der anschließenden pneumatolytisch-hydrothermalen Phase und der dabei stattfindenden Bildung von Beryll, Turmalin, Erzen, Quarzgängen usw., wie auch mit der Bildung von Chlorit, Kaliumfeldspat und Epidot aus Biotit und Sericit, Zeolith, Albit, Calcit, Epidot-Zoisit aus den Feldspäten, gehen die Injektionen zu Ende; natürlich fanden Intrusionen in verschiedenen Stadien statt, so daß Altersunterschiede zwischen den Adern vorhanden sein müssen.

Durch die Einwirkung der hydrothermalen Phase ist manchmal der Biotit im Pegmatit und im Nebengestein in Chlorit und Epidot, manchmal aber auch in Kaliumfeldspat umgewandelt. Ein solches 
Beispiel stellt die Abb. 25 dar. Der ursprüngliche Biotit ist zum Teil in Chlorit, Epidot und Kaliumfeldspat umgewandelt worden.

Der sekundäre, aus Biotit gebildete Kaliumfeldspat ist Mikroklin und zeigt manchmal die für dieses Mineral charakteristische Gitterung und Auslöschungsschiefe auf (001). Der Achsenwinkel $2 \mathrm{~V}_{\alpha}$ schwankt jedoch zwischen $50^{\circ}$ und $68^{\circ}$.

Fraglich bleibt noch die Genese der dritten Gruppe (Plagioklaspegmatite mit basischem Oligoklas bis Andesin und mit nur sehr spärlichen Resten von K-Feldspat). Die Altersbeziehungen konnten durch Gangkreuzungen nicht abgeklärt werden, doch finden sich in der Nähe dieser Pegmatite immer solche der ersten oder zweiten Gruppe. Auf Seite 234 wurde erwähnt, daß man ohne sicheren Beweis vermuten kann, daß diese Pegmatite oft etwas älter sind. Auch die Plagioklaspegmatite lassen hie und da Verdrängungsrelikte eines Kaliumfeldspates erkennen. Ist nun das Vorhandensein von nur kleinen Resten von Kaliumfeldspat ein Beweis dafür, daß dieser Feldspat ursprünglich nur in kleinen Mengen ausgeschieden wurde, oder wurde er eventuell unter Muskovitbildung so stark verdrängt, daß nur kleine Reste von ihm übrig zu bleiben vermochten? Die

Tab. 13. Plagioklase und seltene Mineralien der

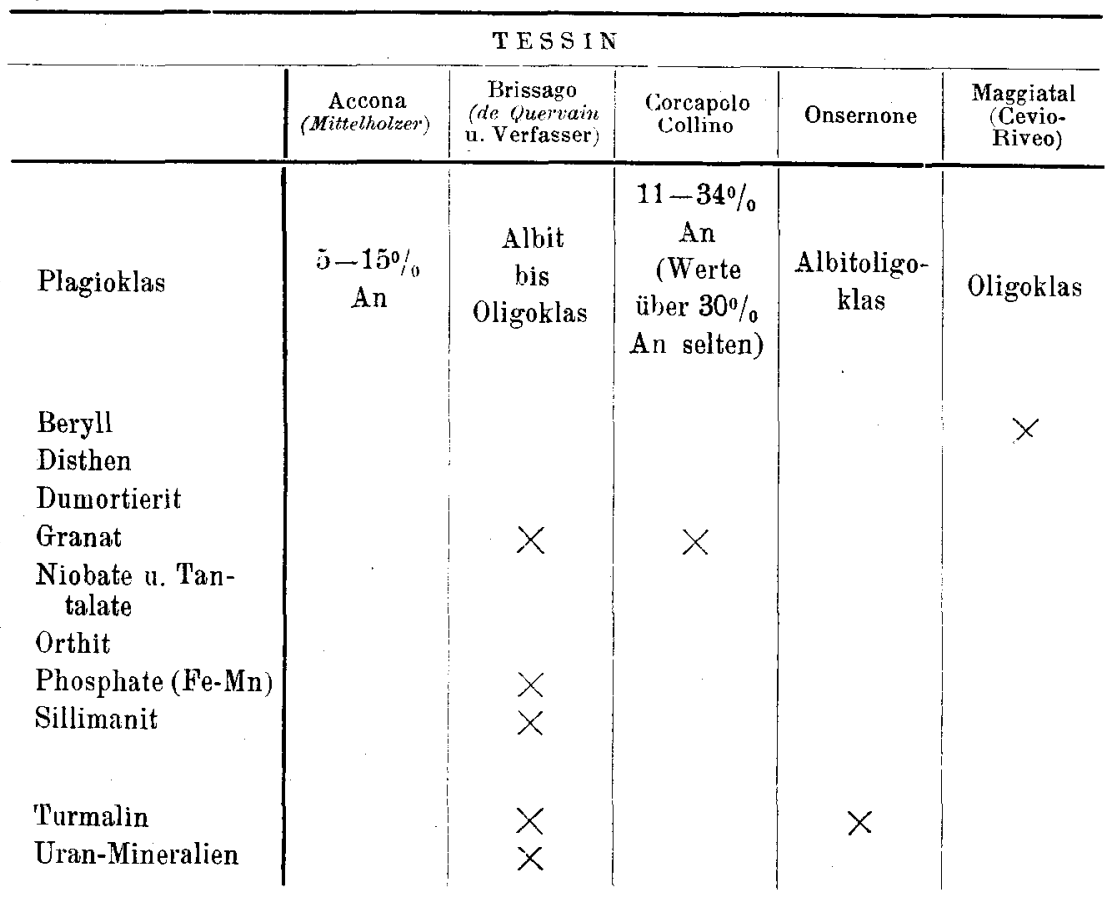


spärlichen Reste des Kaliumfeldspates sind in der Hauptsache im Kristallinnern der Plagioklase gefunden worden, seltener an den Grenzen zwischen den Plagioklaskristallen. Dies spricht zwar zugunsten einer sehr intensiven Verdrängung, doch ist der Plagioklas ziemlich basisch (22 bis $35 \%$ ) und der Biotit überwiegt gegenüber dem Muskovit, so daß eine primäre Ausscheidung von Kaliumfeldspat in großen Mengen kaum anzunehmen ist. So scheint mir am wahrscheinlichsten, daß tatsächlich in diesen Pegmatiten der Kaliumfeldspat primär nicht in großen Mengen vorhanden war und gleichzeitig eine sehr intensive Verdrängung durch den Plagioklas erlitten hatte. Plagioklaspegmatite können auch mit basischen Magmenintrusionen in Beziehung stehen.

Vielleicht dürfen wir dieser Diskussion noch folgendes hinzufügen: Bei Verbano nämlich konnte in den Plagioklaspegmatiten nie Sillimanit gefunden werden, wie das für viele Pegmatitvorkommen der zwei ersten Gruppen dieses Distriktes der Fall ist. Entstammt die Sillimanitbildung (Seite 258) einem Assimilationsvorgang in der Tiefe, so könnte die Abwesenheit des Sillimanites in den Plagioklaspegmatiten entweder die Folge einer Abstammung aus

Tessiner Pegmatite (Zusammenfassang).

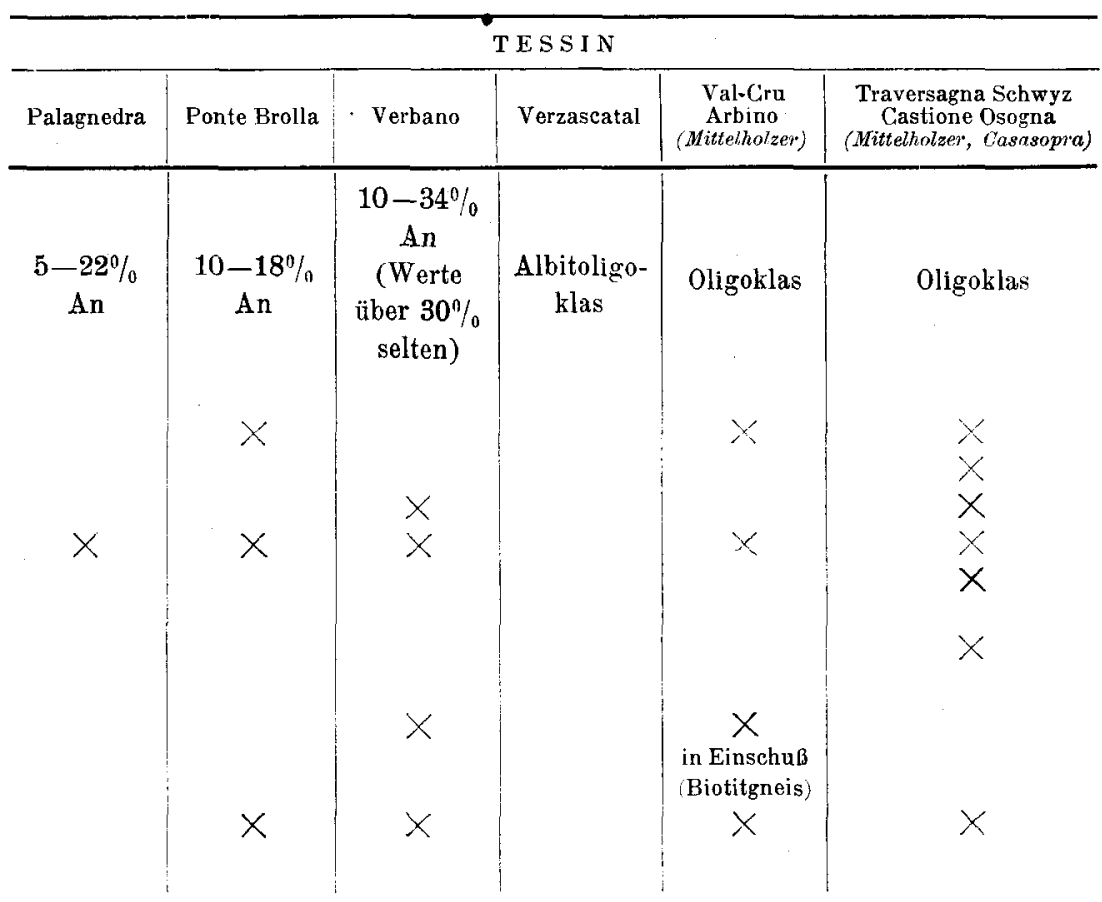


einem anderen Magmaherd sein oder bedeuten, daß die Pegmatite einer älteren Injektion vom gleichen Herd aus vor der Assimilation entstammen. Nun lassen die beschränkte Verbreitung dieser Kategorie von Pegmatiten und die manchmal festgestellte stärkere Verwitterung diese Möglichkeit einer älteren Injektion von einem gemeinsamen Magmaherd aus nicht unwahrscheinlich erscheinen.

\section{Vergleich der Tessiner Pegmatite miteinander und mit denjenigen des Bergells, von Olgiasca am Comersee und Valle Antrona und Ossola im Piemont.}

Beim Vergleich der untersuchten Pegmatite des Tessins untereinander haben wir sowohl den An-Gehalt ihrer Plagioklase als auch die mehr oder weniger sellenen Bestandteile zu berücksichtigen. Über beide Erscheinungen gibt die Tab. 13 Auskunft, in der jedoch die gewöhnlichen Erze, der Zirkon, der Apatit und der Titanit nicht berücksichtigt wurden, weil sie vorwiegend in kleinen Mengen überall auftreten. Um den Vergleich mit den Pegmatiten der Bergells, des Gebieles von Olgiasca am Comersee und der Valle Antrona und Ossola im Piemont zu ermöglichen, wurde die Tab. 14 hinzugefügt.

Tab. 14. Vergleich der Tessiner Pegmatite (Plagioklase und seltene Mineralien) mit denjenigen des Bergells; von Olgiasca am Comersee und Valle Antrona und Ossola im Piemont.

\begin{tabular}{|c|c|c|c|c|}
\hline & Tessin & $\begin{array}{l}\text { Bergell } \\
\text { (Corneitius, } \\
\text { Hugi und } \\
\text { Hir'schi) }\end{array}$ & $\begin{array}{c}\text { Olgiasca, Piona und } \\
\text { Sommafiume (Dervio), } \\
\text { Comersee, Italien. } \\
\text { (Repossi, Cotomelius, } \\
\text { Grill etc.) }\end{array}$ & $\begin{array}{l}\text { Valle Antrona, } \\
\text { Ossola, Piemont, } \\
\text { Italien. (Capitani, } \\
\text { Paglioni, Cossa, } \\
\text { Struever etc.) }\end{array}$ \\
\hline Plagioklas & $\begin{array}{l}\text { Albit u. } \\
\text { Oligoklas } \\
\text { selten } \\
\text { Andesin }\end{array}$ & $\begin{array}{l}\text { Albit u. } \\
\text { Oligoklas. }\end{array}$ & Albit u. Oligoklas & Albit u. Oligoklas \\
\hline Andalusit & & $x$ & & \\
\hline Beryll & $x$ & $x$ & $x$ & $x$ \\
\hline Chrysoberyll & & $x$ & $x$ & $x$ \\
\hline Disthen & $x$ & & & \\
\hline Dumortierit & $x$ & $x$ & & \\
\hline Granat & $x$ & $x$ & $x$ & $x$ \\
\hline $\begin{array}{l}\text { Niobate und } \\
\text { Tantalate }\end{array}$ & $x$ & & & $x$ \\
\hline Orthit & $x$ & $x$ & & \\
\hline Phosphate (Fe-Mn) & $x$ & & $x$ & \\
\hline Sillimanit & $x$ & $x$ & & \\
\hline Turmalin & $x$ & $x$ & $x$ & $x$ \\
\hline Uran-Mineralien & $x$ & $x$ & $x$ & $x$ \\
\hline
\end{tabular}

